\title{
PHYSICS OF THE PHASE CHANGES OF Be STARS
}

\author{
K.K. GHOSH \\ Indian Institute of Astrophysics, Vainu Bappu Observatory, Kavalur, India
}

\begin{abstract}
Based on spectroscopic observations of more than 100 Be stars, we present the results of the phase changes of Be stars.
\end{abstract}

\section{Introduction}

Be stars display $\mathrm{B}, \mathrm{Be}$ and Be shell spectra and the cause of the phase changes is mostly unknown. Kogure (1990) has suggested a simple model of phase-changing variation between Be stars and shell stars from the viewpoint of the formation of the shell absorption lines in the envelopes of these stars. Here we present the observational results which are directly connected with the phase-change variations of Be stars.

\section{Observations}

We have started a spectroscopic program, since 1988, at the Cassegrain and Coudé foci of the $2.34 \mathrm{~m}$ and $1 \mathrm{~m}$ reflectors of VBO, for faint (brighter than $14 \mathrm{mag}$ ) and bright (brighter than $7 \mathrm{mag}$ ) Be stars, respectively. Using the Coudé Echelle spectrograph with CCD system we get the spectra, simultaneously, in the He I (4471 $\AA), \mathrm{Mg}$ II $(4481 \AA), \mathrm{H} \beta(4861 \AA)$, He I (5876 $\AA)$, Si II $(6374 \& 6371 \AA)$ and $H$ regions. From the obtained spectra we have detected phase change variations in many Be stars ( $\gamma$ Cas, $\phi$ Per, HR 1423, $27 \mathrm{CMa}, \lambda$ Eri, FY CMa, $\mu$ Cen, 48 Lib, etc.).

\section{Results and discussion}

We have found that the phase-change variations of Be stars from $\mathrm{Be}$ to Be-shell phase take place via a strong outburst with $P$ Cygni profiles in their spectra (in Figs. 1-3, the P-Cygni profiles of FY CMa during February 1991 have just been mentioned (Peters 1991, Ghosh et al. 1993). Before the outburst the $\mathrm{H} \beta$ and He I ( $5876 \AA$ ) lines displayed inverse P Cygni profiles during March 1990. Also we have detected inverse P-Cygni profiles in the He I (5876 $\AA$ ) and $\mathrm{H} \beta$ lines of many other Be stars before their outburst (Ghosh 1990, Ghosh et al. 1993). Inverse P-Cygni profiles indicate the infall of matter on the star and this may cause the instability of the star which leads to the outburst. The amount of material ejected due to the outburst is much larger then that ejected by the mass loss due to the non-radial pulsation of the star (Ghosh et al. 1993). After the outburst the star enters 
into the Be shell phase (see Figs. 1-3, the shell profiles of FY CMa during 29 March 1991). After some time the envelope of the shelter star disperses into the interstellar medium (ISM) and the star enters either into a B phase (if there is no new mass loss between the outburst and the disappearance of the envelope into the ISM) or into Be phase (if there is new mass loss from the star).

\section{Conclusion}

Phase-change variations of $\mathrm{Be}$ stars from the $\mathrm{B}$ to $\mathrm{Be}$ phase take place via mass loss of the star due to its non-radial pulsations (Baade 1989) and from the Be to Be-shell phase is due to the disappearance of the shell envelope into the ISM when no new mass loss occurs between the outburst and the disappearance of the envelope. If there is new mass loss, the star will enter into Be phase from the Be-shell phase (see Fig. 4).

\section{References}

Baade, D.: 1989, Astronomy and Astrophysics 198, 211

Ghosh, K.K.: 1990, Astrophysical Journal 360, 239

Ghosh, K.K. et al., 1993, in preparation

Kogure, T.: 1990, Astrophysics and Space Science 193, 7

Peters, G.J.: 1991, Be Star News Lett. 24, 17

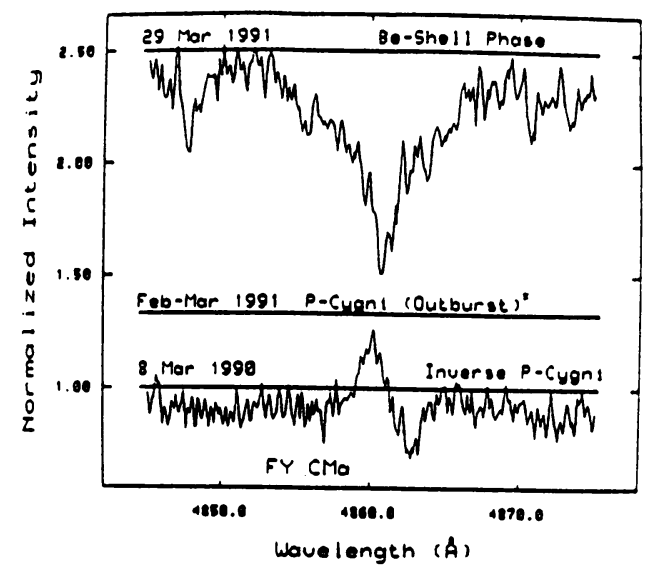

Fig. 1. $\mathrm{H} \beta$ profiles of FY CMa at different phases. 


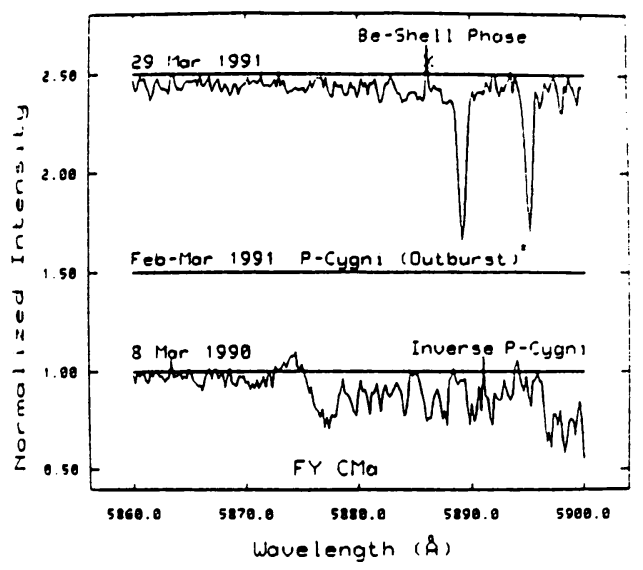

Fig. 2. Same as Fig. 1 but for He I profiles

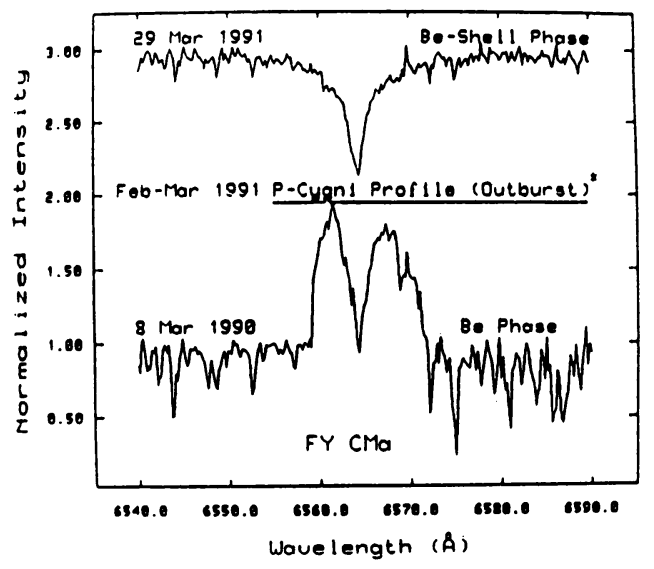

Fig. 3. Same as Fig. 1 but for $\mathrm{H} \alpha$ profiles

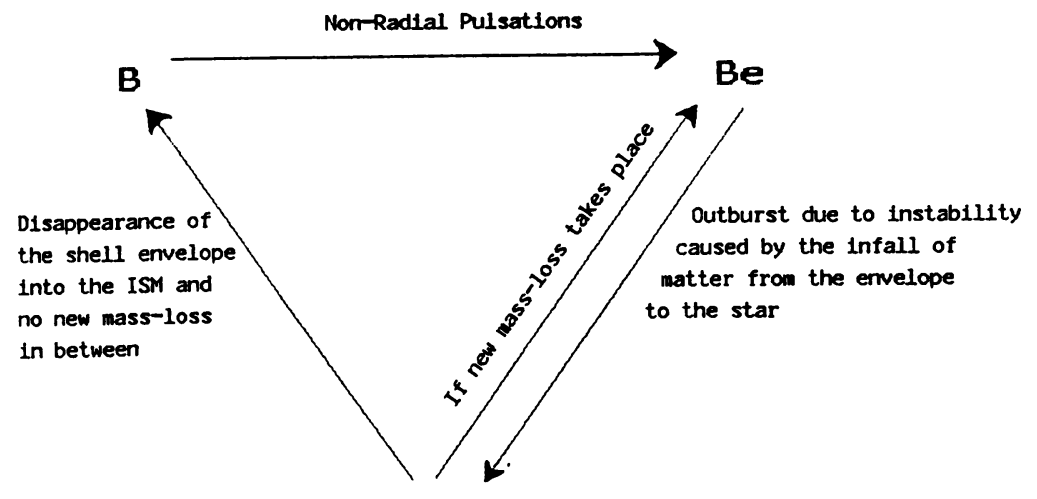

Be - Shell

Fig. 4. Schematic diagram of the phase-change variations of Be stars. 